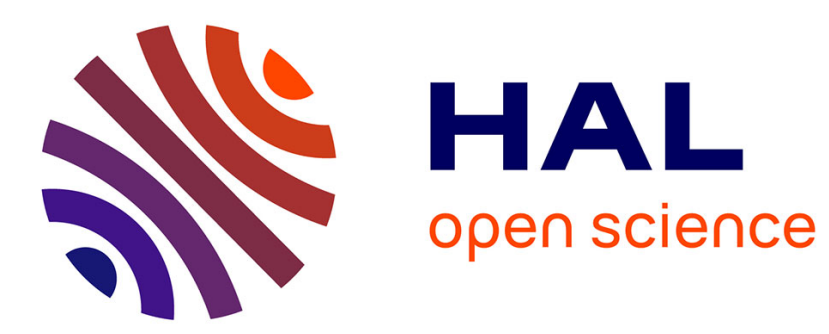

\title{
Dislocations d'interface et défauts de volume dans l'hétérostructure GaSb/GaAs
}

\author{
A. Rocher, F.W.O. da Silva, C. Raisin
}

\section{To cite this version:}

A. Rocher, F.W.O. da Silva, C. Raisin. Dislocations d'interface et défauts de volume dans l'hétérostructure GaSb/GaAs. Revue de Physique Appliquée, 1990, 25 (9), pp.957-961. 10.1051/rphysap:01990002509095700 . jpa-00246263

\section{HAL Id: jpa-00246263 https://hal.science/jpa-00246263}

Submitted on 1 Jan 1990

HAL is a multi-disciplinary open access archive for the deposit and dissemination of scientific research documents, whether they are published or not. The documents may come from teaching and research institutions in France or abroad, or from public or private research centers.
L'archive ouverte pluridisciplinaire HAL, est destinée au dépôt et à la diffusion de documents scientifiques de niveau recherche, publiés ou non, émanant des établissements d'enseignement et de recherche français ou étrangers, des laboratoires publics ou privés. 
Classification

Physics Abstracts

$61.16-61.70-68.00-81.15$

\title{
Dislocations d'interface et défauts de volume dans l'hétérostructure GaSb/GaAs
}

\author{
A. Rocher, F. W. O. Da Silva $\left({ }^{1}\right)$ et C. Raisin $\left({ }^{1}\right)$ \\ Centre d'Elaboration des Matériaux et d'Etudes Structurales, CEMES/LOE du CNRS, 29 rue Jeanne Marvig, \\ 31400 Toulouse, France
}

(1) LESIC, USTL, Place E. Bataillon, 34060 Montpellier, France

(Reçu le 21 décembre 1989, accepté le 26 février 1990)

\begin{abstract}
Résumé. - Du GaSb a été déposé sur du GaAs par épitaxie par jets moléculaires à $470{ }^{\circ} \mathrm{C}$. A cette température la croissance est tridimensionnelle dans les premiers stades de l'épitaxie. Après coalescence des îlots de $\mathrm{GaSb}$, la croissance devient bidimensionnelle. Les dislocations liées au désaccord paramétrique de $8 \%$ sont toutes de type Lomer. Elles sont organisées en réseau périodique à deux dimensions. La très faible densité de défauts expérimentalement observée est liée au nombre réduit d'imperfections qui existent au niveau du système de dislocations d'interface.
\end{abstract}

\begin{abstract}
GaSb on $\mathrm{GaAs}$ has been grown by Molecular Beam Epitaxy at $470{ }^{\circ} \mathrm{C}$. At this temperature a 3dimensional growth mode occurs at the first stage of the epitaxy. After $\mathrm{GaSb}$ island coalescence the growth mode becomes 2-dimensional. Misfit dislocations, due to a $8 \%$ lattice mismatch, are Lomer type. The misfit dislocation network is well organized as a regular array. The very low density of threading defects is related to the low number of imperfections of this misfit dislocation network.
\end{abstract}

\section{Introduction.}

Les composants électroniques ont leurs propriétés spécifiques très perturbées par les défauts de fabrication créés en particulier lors de la croissance par épitaxie de la couche III-V. Si l'origine de ces défauts - la contrainte associée au désaccord paramétrique - est bien établie, la réduction de leur densité constitue une question qui n'a pas encore trouvé de réponse satisfaisante [1]. En fait, le véritable problème de ces défauts n'est pas de chercher à les annihiler mais plutôt de trouver comment ne pas les créer. A partir de cette idée, il est nécessaire de comprendre les mécanismes de création de ces défauts en relation avec le mode de croissance par épitaxie.

Cet article présente nos premiers résultats concernant les défauts associés à l'hétérostructure $\mathrm{GaSb} / \mathrm{GaAs}$. Son objet est de montrer que malgré ses $8 \%$ de désaccord paramétrique la structure $\mathrm{GaSb} / \mathrm{GaAs}$ présente, dans des conditions favorables de croissance, un système de dislocations d'interface remarquablement bien organisé.

\section{Croissance du GaSb/GaAs.}

L'idée d'étudier le système $\mathrm{GaSb} / \mathrm{GaAs}$ correspondait à l'espoir d'obtenir un réseau de dislocation idéal en relation avec la surface de substrat parfaite obtenue à partir d'une couche tampon de GaAs. De plus, il nous paraissait intéressant, pour minimiser la densité de défauts en volume, de relaxer les contraintes du dépôt le mieux et le plutôt possible. Pour cela, au lieu de suivre les idées conventionnelles de la croissance bidimensionnelle, nous avons choisi de travailler à la température de l'homoépitaxie du $\mathrm{GaSb}$.

Les échantillons ont été réalisés dans un bâti d'épitaxie par jets moléculaires (EJM) qui a été décrit en détail par C. Raisin et al. [2]. L'enceinte de croissance comporte des cellules à effusion de $\mathrm{Ga}$, As et Sb. Elle est équipée d'un dispositif de diffrac- 
tion d'électrons en incidence rasante (RHEED à $10 \mathrm{kV}$ ) qui permet de suivre la qualité de la surface pendant la croissance. Un équipement d'analyse Auger est installé dans une autre enceinte où les échantillons peuvent être transférés sous vide pour une caractérisation chimique de surface.

Les échantillons sont préparés de la façon suivante :

- un substrat de GaAs orienté (001) est chauffé à une température de $600{ }^{\circ} \mathrm{C}$ sous flux d'arsenic pour obtenir une surface propre et ordonnée;

- une couche tampon de $1 \mu \mathrm{m}$ de GaAs est déposée par EJM à $580{ }^{\circ} \mathrm{C}$. Elle donne un très bon état de surface dont la qualité a été évaluée par RHEED ;

- après un arrêt de croissance nécessaire pour stopper le flux d'arsenic, le GaSb est déposé par EJM à $470{ }^{\circ} \mathrm{C}$, température classique d'homoépitaxie du GaSb.

Les vitesses de croissance du GaAs et du GaSb sont de l'ordre de $0,7 \mu$ /heure.

Des dépôts de $77 \AA$ et $770 \AA$ de GaSb ont été réalisés pour étudier le mode de croissance, les dislocations d'interface et les défauts de volume. L'épaisseur de $770 \AA$ est déjà représentative de l'état massif pour le comportement des défauts de volume.

\section{Méthode d'observations.}

Pour mettre en évidence l'origine des défauts de volume, nous avons été amenés à étudier par microscopie électronique en transmission le système de dislocations d'interface dans son ensemble. Les observations d'hétérostructures sur la tranche (cross-section) ne répondent pas à cet objectif car l'information fournie ne porte que sur une dimension: seules les dislocations parallèles au faisceau d'électrons sont observées en projection. Par contre, les vues planes, où le faisceau d'électrons est parallèle à la direction de croissance, permettent de voir en projection directe l'ensemble de ce système [3].

Les observations ont été faites par microscopie électronique en transmission à $200 \mathrm{kV}$ en vue plane sur des échantillons d'épaisseur inférieure à $1500 \AA$ qui sont transparents aux électrons. Leur préparation se fait sans toucher au dépôt de GaSb par amincissement chimique du substrat de GaAs par la face arrière.

Les dislocations d'interface, qui se trouvent à moins de $50 \AA$ les unes des autres, ne sont observables que par la technique classique du faisceau faible en fond noir [4].

\section{Résultats expérimentaux.}

4.1 MORPHOLOGIE DES DÉPÔTS DE GaSb. - La figure 1 est une micrographie en champ clair qui

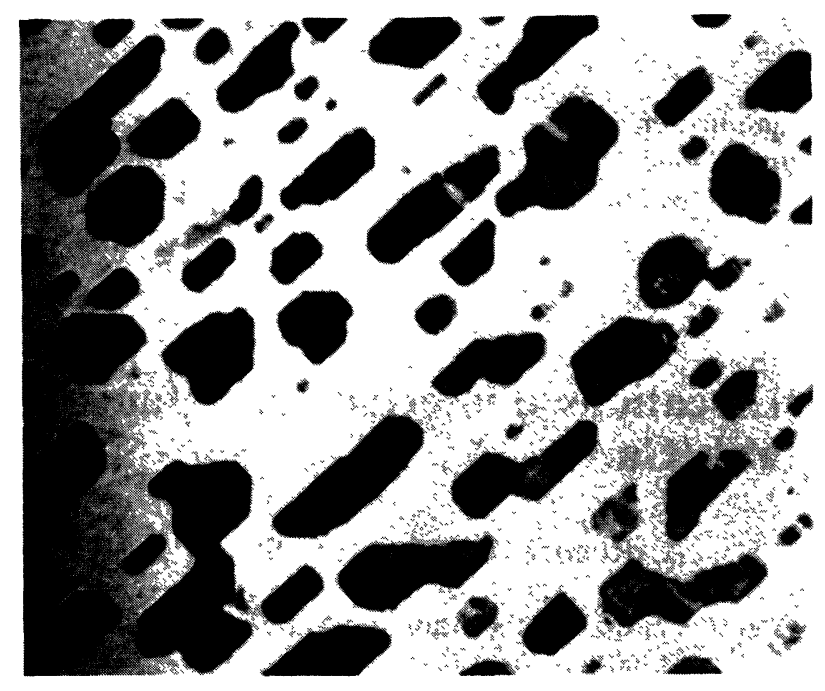

Fig. 1. - Micrographie en vue plane montrant la nucléation hétérogène du GaSb déposé par EJM sur GaAs.

[Plan view TEM micrograph showing the heterogeneous nucleation of $\mathrm{GaSb}$ layers on GaAs.]

montre la morphologie du dépôt de $77 \AA$. Les contrastes sombres sont directement liés à la présence de $\mathrm{GaSb}$. Les régions claires en sont exemptes. Le dépôt se présente sous forme d'îlots avec un taux de couverture compris entre 35 et $40 \%$. Ces îlots ont deux tailles différentes: les plus petits ont des dimensions inférieures à $1000 \AA$; les plus grands ont des formes allongées avec des dimensions de l'ordre de $0.1 \mu \mathrm{m} * 1 \mu \mathrm{m}$. La forme des îlots est définie dans le plan de base (001) par des directions cristallographiques $\langle 110\rangle$ et $\langle 100\rangle$. Les îlots les plus grands ont des formes allongées suivant la direction [1-10]. La dissymétrie de forme pourrait être liée à l'anisotropie de diffusion des atomes de gallium dans les directions [110] et [1-10] du plan (001) [5].

Des observations faites en cross-section sur le dépôt de $77 \AA$ montrent que l'épaisseur déposée est de l'ordre de $200 \AA$ pour les îlots dont la taille est supérieure à $1000 \AA$ de côté. Cete valeur est tout à fait cohérente avec le taux de couverture qui a été mesuré égal à environ $35 \%$. Ce résultat indique que le dépôt de $\mathrm{GaSb}$ se fait principalement par développement latéral des îlots suivant des plans $\{111\}$. Ce mode de croissance en îlots doit être relié à une vitesse de croissance suivant les plans $\{111\}$ supérieure à celle suivant le plan (001).

La figure 2 présente le réseau de dislocations d'interface observé pour un îlot isolé. Le réseau de dislocations n'est pas parfait. Il contient au moins un défaut qui semble être à l'origine de la nucléation du $\mathrm{GaSb}$.

Le dépôt de $770 \AA$ est constitué d'une couche d'épaisseur constante. L'analyse Auger montre que 


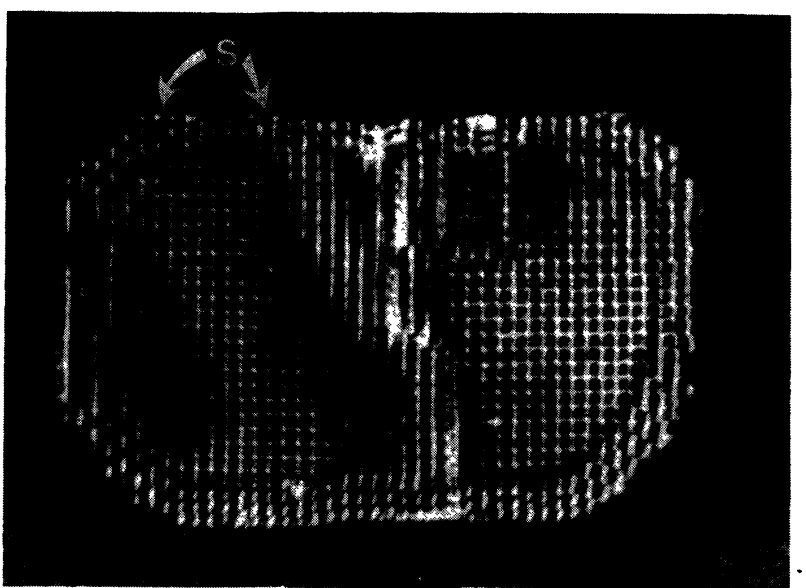

Fig. 2. - Ilot de GaSb/GaAs : observation simultanée des deux familles de dislocations d'interface. Le réseau n'est pas parfait, il contient des défauts. Noter en $S$ le développement d'une dislocation de Lomer sous forme de segments limités en bord d'îlot.

[GaSb hillock: simultaneous observation of the two families of interfacial dislocations. The dislocation network is not perfect and contains some defects. Note that the Lomer dislocation segments, arrowed S, are created at the limit of the hillock.]

le taux de couverture est égal à 1 lorsque l'épaisseur déposée atteint $500 \AA \AA$. A partir de là les observations en RHEED font apparaître que l'épaisseur déposée est de plus en plus uniforme et donc que la croissance devient de plus en plus bidimensionnelle.

4.2 Dislocations D’interface. - Ces dislocations rattrapent le désaccord paramétrique. Elles sont nécessaires à la stabilité de la structure et sont, dans le cas idéal, organisées en deux réseaux parallèles aux directions $\langle 110\rangle$ du plan d'interface.

La figure 3 est une micrographie réalisée en faisceau faible avec un plan réflecteur (220). Elle ne fait apparaître qu'une des deux familles de dislocations alignées suivant une direction $\langle 110\rangle$. L'autre famille est hors contraste. Le plan réflecteur (2-20) donne une image équivalente de l'autre famille de dislocations. Ces images permettent de caractériser ces dislocations par leur direction $u$ et leur vecteur de Burgers $b$ :

$$
\begin{array}{lll}
u=[110] & \text { et } & b=1 / 2[1-10] \\
u=[1-10] & \text { et } & b=1 / 2[110]
\end{array} .
$$

Ces deux familles de dislocations se trouvent donc localisées dans le plan (001) de l'interface. L'espacement entre dislocations, mesuré sur la figure 3 , est égal à $50 \AA$ pour les deux familles. Cette valeur correspond exactement à celle prévue par la relation qui donne la distance entre dislocations en fonction du désaccord paramétrique $f$ et de la composante efficace de rattrapage de leur vecteur de Burgers $b$ :

$$
D=b / f=5,64 /(\sqrt{2} \times 0,08)=50 \AA .
$$

Ces deux résultats, cohérents entre eux, conduisent à la même conclusion : toutes les dislocations qui rattrapent normalement le désaccord paramétrique entre le GaAs et le $\mathrm{GaSb}$ sont de type coin, dit de Lomer. Compte tenu du désaccord de maille de $8 \%$, la longueur de dislocation nécessaire à l'adaptation de deux mailles cristallines est d'environ $40 \mathrm{~km}$ par $\mathrm{cm}^{2}$ d'interface.

$\mathrm{La}$ régularité du système de dislocations d'interface observée sur la figure 3 conduit à penser que sa création est un mécanisme simple et bien défini qui minimise fortement l'énergie du système. La création des dislocations d'interface ne semble pas reliée à un mécanisme conventionnel de source de dislocations qui glisseraient suivant des plans $\{111\}$ à partir de la surface libre (001) du GaSb. Ces dislocations ont plutôt une origine qui est en relation directe avec la forme des îlots de GaSb.

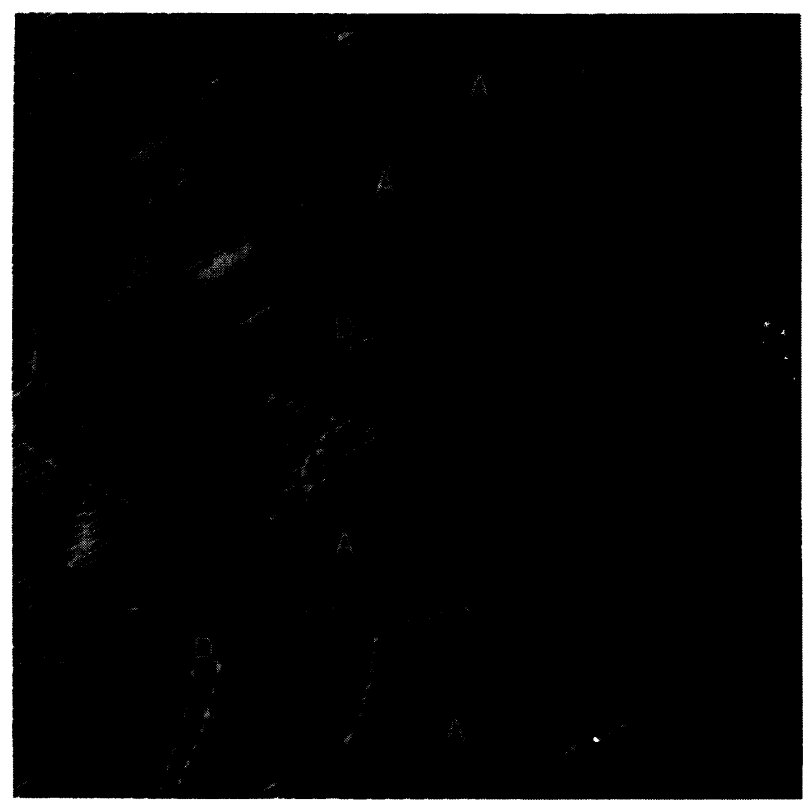

Fig. 3. - Dislocations d'interface : observation d'une des deux familles de dislocations réalisée avec le plan réflecteur $\{220\}$. L'espacement entre dislocations et les conditions d'extinction sont caractéristiques du caractère coin de ces dislocations. Noter les discontinuités des dislocations en $\mathrm{A}$. Les défauts de volume, repérés en $\mathrm{D}$, ont pour origine des imperfections du système de dislocations d'interface.

[Interfacial dislocations observed in plan view. Only one family is in contrast. Both their $50 \AA$ spacing and extinction conditions characterize the edge type of interfacial dislocations. Note the dislocation discontinuities, arrowed A. Threading defects, arrowed D, are related to the dislocation network imperfections.] 
Les dislocations semblent se créer progressivement au fur et à mesure de la croissance de l'îlot de $\mathrm{GaSb}$ par développement des faces $\{111\}$ comme cela est schématisé sur la figure 4. L'observation sur la figure 2 de segments de dislocation discontinus en bord d'îlot irait dans ce sens. Toutefois le mécanisme de création directe de ces dislocations de Lomer reste encore à établir.

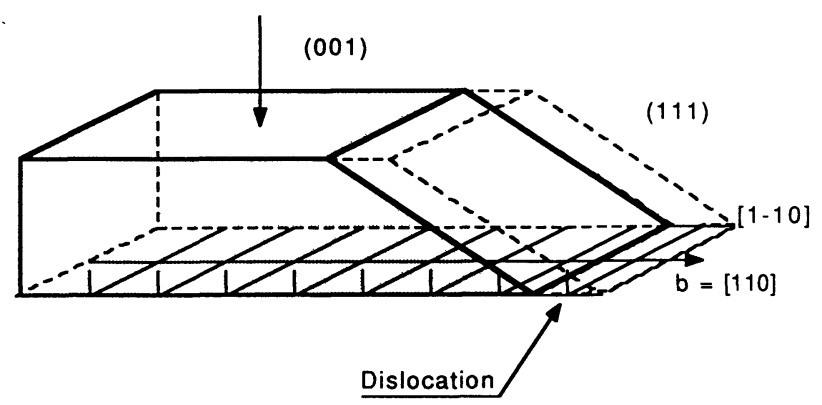

Fig. 4. - Schéma montrant une origine possible des dislocations d'interface.

[Schema showing a possible origin of the interfacial Lomer dislocations.]

4.3 ORIGINE DES DÉFAUTS DE VOLUME. - La coalescence entre îlots est admise comme étant la principale source de défauts susceptibles de se propager dans la couche épitaxiée. Ici, les dislocations d'interface, nécessaires à la stabilité de l'hétérostructure $\mathrm{GaSb} / \mathrm{GaAs}$ sont organisées en réseaux de tailles limitées. La jonction entre ces sousréseaux se fait avec ou sans émission de défauts.

Le réseau de dislocations d'interface est constitué de sous-réseaux dont l'origine est liée à la croissance en îlots. La jonction entre ces sous-réseaux se présente sous forme de discontinuités qui sont caractérisées par un décalage d'un demi-espacement (25 ̊) entre les dislocations comme cela est observé sur la figure 3 en A. Il n'y a en général pas de défaut émis au niveau de ces discontinuités. La coalescence entre ilôts se fait donc ici sans émission de défaut dans le volume. Il conviendrait d'établir la réaction qui intervient pour créer ces discontinuités.

Les dislocations de volume observées sur la figure 3 sont toutes isolées. Elles proviennent d'imperfections du réseau de dislocations d'interface. Une imperfection typique est observée sur la figure 3 en $\mathrm{D}$ : une dislocation de volume est créée à partir d'une dislocation d'interface qui s'est courbée.

La densité de ces dislocations de volume au voisinage de l'interface est évaluée sur la figure 3 .
Elle est de l'ordre de $10^{+10} / \mathrm{cm}^{2}$. Cette valeur est à comparer à celle du GaAs/Si où la mesure n'est pas réellement possible car les défauts ne sont pas séparés [6]. Pour la plupart des hétérostructures qui croissent dans de bonnes conditions la qualité cristalline de la couche s'améliore avec l'épaisseur du dépôt. Ainsi dans le GaAs/Si la densité superficielle de dislocations expérimentalement mesurée est de l'ordre de $10^{+8} / \mathrm{cm}^{2}$ après un dépôt de $3 \mu \mathrm{m}$ de GaAs.

\section{Discussion et conclusion.}

La densité des défauts de volume dans les couches déposées par épitaxie est directement fonction des imperfections du système de dislocations d'interface associé au désaccord de paramètre cristallin entre le $\mathrm{GaSb}$ et le GaAs. La qualité de ce système dépend surtout de l'état de surface du substrat et du mode de croissance du matériau déposé plus que du désaccord paramétrique $f$.

La perfection de la surface du substrat de GaAs est certainement un facteur important qui permet d'obtenir une couche $\mathrm{GaSb}$ présentant une bonne qualité cristalline. L'utilisation de couche tampon répond en grande partie à ce problème. Le dépôt de $\mathrm{GaSb}$ sera d'autant plus parfait que la croissance sera faite à une température telle que les contraintes associées au désaccord paramétrique pourront complètement relaxer. La température de $470{ }^{\circ} \mathrm{C}$, utilisée pour l'homoépitaxie de $\mathrm{GaSb}$, facilite cette relaxation. Les premiers stades de croissance du $\mathrm{GaSb}$ se font, dans ces conditions, de façon tridimensionnelle. Ils favorisent la relaxation des contraintes par un système organisé de dislocation à l'interface. Enfin, lorsque la coalescence des îlots est totale la croissance devient progressivement bidimensionnelle. L'épaisseur uniforme du dépôt semble atteinte pour des dépôts de $500 \AA$.

Ainsi, contrairement à l'idée couramment admise, la présence de dislocations dans ces systèmes peut constituer un facteur de stabilité des dispositifs. En particulier, si ces dislocations s'organisent dans le plan de l'interface en un réseau régulier qui relaxe complètement les contraintes, il n'y aura pas de défauts étendus dans la couche déposée. Le problème est alors de forcer la nature à réaliser directement des réseaux de dislocations les plus parfaits possibles.

\section{Remerciements.}

Nous remercions vivement Monsieur Jacques Crestou pour l'excellente préparation des échantillons destinés aux observations en microscopie électronique en transmission. 


\section{Bibliographie}

[1] a) «III-V heterostructures for electronic/photonic devices ", Mat. Res. Soc. Symp. Proc. 145, C. W. Tu, V. D. Mattera Jr, A. C. Gossart Eds. (1989);

b) « Chemistry and defects in semiconductor heterostructures ", Mat. Res. Soc. Symp. Proc., 148, M. Kawabe, E. R. Weber, R. D. Sands \& R. S. Williams Eds. (1989).

[2] Raisin C., Saguintaah B., Tegmousse H., Lassabatere L., Girault B. et Alibert C., Ann. Telecommun. 41 (1986) 50.
[3] Rocher A. et Charasse M. N., dans Gettering and Defect Engineering in the Semiconductor Technology. Ed. M. Kittler, Sci. Tech. Publ. (1989) p. 547.

[4] Edington J. W., Pract. Electron Microsc. Mater. Sci. 3 (Mc Millan Press ltd) 1975.

[5] MASSIES J., communication privée.

[6] Rocher A., Charasse M. N., Bartenlian B. et Chazelas J., Proc. of the International Conference on «Interphases and Intergranular Boundaries in materials ", Editions de Physique, mars 1990. 